\title{
Soft-gluon corrections to hard-scattering cross sections through NNNLO
}

\author{
Nikolaos Kidonakis* \\ Kennesaw State University, Physics \#1202, Kennesaw, GA 30144-5591, USA \\ E-mail: nkidonak@kennesaw.edu
}

\begin{abstract}
I present a systematic approach to calculating soft-gluon corrections through next-to-next-to-nextto-leading order for arbitrary hard-scattering cross sections. Using a unified approach, master formulas are derived for processes with both simple and complex color flows. Applications and numerical results are given for some processes of interest, including charged Higgs production and top quark production in the Standard Model and beyond.
\end{abstract}

International Europhysics Conference on High Energy Physics

July 21st - 27th 2005

Lisboa, Portugal

${ }^{*}$ Speaker. 


\section{Soft-gluon corrections}

Physical cross sections can be calculated in perturbative QCD [1] as convolutions of perturbative hard-scattering cross sections $\hat{\sigma}$ with non-perturbative parton distribution functions $\phi$. Near threshold for the production of a specified system there is restricted phase space for real gluon emission. This results in an incomplete cancellation of infrared divergences between real and virtual graphs, which gives rise to large logarithms. If we define $s_{4}=s+t_{1}+t_{2}-\sum m^{2}$, where the $t_{i}$ are standard kinematical invariants and we sum over the particle masses, then $s_{4} \rightarrow 0$ at threshold and the soft and collinear corrections take the form of plus distributions $\mathscr{D}_{l}\left(s_{4}\right) \equiv\left[\left(\ln ^{l}\left(s_{4} / M^{2}\right)\right) / s_{4}\right]_{+}$ with $M$ a relevant hard scale and $l \leq 2 n-1$ for the $n$-th order corrections. The terms with $l=2 n-1$ are the leading logarithms (LL), those with $l=2 n-2$ are the next-to-leading logarithms (NLL), and so on $[2,3]$. These corrections exponentiate in moment space. If we define moments of the cross section by $\hat{\sigma}(N)=\int_{0}^{\infty} d s_{4} e^{-N s_{4} / M^{2}} \hat{\sigma}\left(s_{4}\right)$ then the soft corrections become $\mathscr{D}_{l}\left(s_{4}\right) \rightarrow \frac{(-1)^{l+1}}{l+1} \ln ^{l+1} N+\cdots$ We can formally resum these logarithms to all orders in $\alpha_{s}$ by factorizing the soft gluons from the hard scattering. To invert a resummed cross section back to momentum space one needs to employ a prescription to deal with the Landau pole, and this creates unavoidable ambiguities. However if one expands the resummed cross section at any fixed order, no matter how high, then the inversion can be done without resorting to prescriptions [4].

At next-to-leading order (NLO) in $\alpha_{s}$ we have $\mathscr{D}_{1}\left(s_{4}\right)(\mathrm{LL})$ and $\mathscr{D}_{0}\left(s_{4}\right)$ (NLL) terms. At nextto-next-to-leading order (NNLO) we have $\mathscr{D}_{3}\left(s_{4}\right)(\mathrm{LL}), \mathscr{D}_{2}\left(s_{4}\right)(\mathrm{NLL}), \mathscr{D}_{1}\left(s_{4}\right)(\mathrm{NNLL})$, and $\mathscr{D}_{0}\left(s_{4}\right)$ (NNNLL) terms. At next-to-next-to-next-to-leading order (NNNLO) we have $\mathscr{D}_{5}\left(s_{4}\right)(\mathrm{LL}), \mathscr{D}_{4}\left(s_{4}\right)$ $(\mathrm{NLL}), \mathscr{D}_{3}\left(s_{4}\right)(\mathrm{NNLL}), \mathscr{D}_{2}\left(s_{4}\right)(\mathrm{NNNLL}), \mathscr{D}_{1}\left(s_{4}\right)$ (NNNNLL), and $\mathscr{D}_{0}\left(s_{4}\right)$ (NNNNNLL) terms.

Threshold resummation has by now been applied to a large number of processes, most recently to top quark pair hadroproduction [5], charged Higgs production [6], large- $Q_{T} W$ [7] and Higgs [8] production, and FCNC top production [9]. The numerical results typically show that the higherorder corrections are sizable and they dramatically decrease the scale dependence.

\section{NNNLO corrections}

A unified expression for the resummed cross section for an arbitrary process is given by $[4,10]$

$$
\begin{aligned}
\hat{\sigma}^{r e s}(N)= & \exp \left[\sum_{i} E^{f_{i}}\left(N_{i}\right)+E_{\text {scale }}^{f_{i}}\left(\mu_{F}, \mu_{R}\right)\right] \exp \left[\sum_{j} E^{\prime f_{j}}\left(N_{j}\right)\right] \\
& \times \operatorname{Tr}\left\{H^{f_{i} f_{j}} \exp \left[\int \frac{d \mu^{\prime}}{\mu^{\prime}} \Gamma_{S}^{\dagger} f_{i} f_{j}\right] S^{f_{i} f_{j}} \exp \left[\int \frac{d \mu^{\prime}}{\mu^{\prime}} \Gamma_{S}^{f_{i} f_{j}}\right]\right\},
\end{aligned}
$$

where $\mu_{F}$ is the factorization scale and $\mu_{R}$ is the renormalization scale. The exponents $E^{f_{i}}$ and $E^{\prime f_{j}}$ resum contributions from incoming and outgoing partons, respectively, $H$ are hard-scattering matrices in color space, and $S$ are soft matrices that describe noncollinear soft-gluon emission and whose evolution is given in terms of the soft anomalous dimension matrices $\Gamma_{S}$. See Refs. $[4,10]$ for details. 
The expansion of the resummed cross section to NLO provides us with a master formula for the NLO soft-gluon corrections

$$
\hat{\sigma}^{(1)}=\sigma^{B} \frac{\alpha_{s}\left(\mu_{R}^{2}\right)}{\pi}\left\{c_{3} \mathscr{D}_{1}\left(s_{4}\right)+c_{2} \mathscr{D}_{0}\left(s_{4}\right)+c_{1} \delta\left(s_{4}\right)\right\}+\frac{\alpha_{s}^{d_{\alpha_{s}}+1}\left(\mu_{R}^{2}\right)}{\pi}\left[A^{c} \mathscr{D}_{0}\left(s_{4}\right)+T_{1}^{c} \delta\left(s_{4}\right)\right]
$$

with $c_{3}=\sum_{i} 2 C_{i}-\sum_{j} C_{j}$, where for quarks $C_{q}=C_{F}=\left(N_{c}^{2}-1\right) /\left(2 N_{c}\right)$ and for gluons $C_{g}=C_{A}=N_{c}$. Also $c_{2}=c_{2}^{\mu}+T_{2}$, with $c_{2}^{\mu}=-\sum_{i} C_{i} \ln \left(\mu_{F}^{2} / M^{2}\right)$ and $T_{2}=-\sum_{i}\left[C_{i}+2 C_{i} \ln \left(-t_{i} / M^{2}\right)+C_{i} \ln \left(M^{2} / s\right)\right]-$ $\sum_{j}\left[B_{j}^{(1)}+C_{j}+C_{j} \ln \left(M^{2} / s\right)\right], A^{c}=\operatorname{tr}\left(H^{(0)} \Gamma_{S}^{\prime(1) \dagger} S^{(0)}+H^{(0)} S^{(0)} \Gamma_{S}^{(1)}\right)$, and $c_{1}=c_{1}^{\mu}+T_{1}$, with $c_{1}^{\mu}=$ $\sum_{i}\left[C_{i} \ln \left(-t_{i} / M^{2}\right)-\gamma_{i}^{(1)}\right] \ln \left(\mu_{F}^{2} / M^{2}\right)+d_{\alpha_{s}}\left(\beta_{0} / 4\right) \ln \left(\mu_{R}^{2} / M^{2}\right)$, where $B_{q}^{(1)}=\gamma_{q}^{(1)}=3 C_{F} / 4$ and $B_{g}^{(1)}=$ $\gamma_{g}^{(1)}=\beta_{0} / 4$.

The master formula for the NNLO soft-gluon corrections is

$$
\begin{aligned}
\hat{\sigma}^{(2)}=\sigma^{B} \frac{\alpha_{s}^{2}\left(\mu_{R}^{2}\right)}{\pi^{2}} \frac{1}{2} c_{3}^{2} \mathscr{D}_{3}\left(s_{4}\right) \\
+\sigma^{B} \frac{\alpha_{s}^{2}\left(\mu_{R}^{2}\right)}{\pi^{2}}\left\{\frac{3}{2} c_{3} c_{2}-\frac{\beta_{0}}{4} c_{3}+\sum_{j} C_{j} \frac{\beta_{0}}{8}\right\} \mathscr{D}_{2}\left(s_{4}\right)+\frac{\alpha_{s}^{d_{\alpha_{s}}+2}\left(\mu_{R}^{2}\right)}{\pi^{2}} \frac{3}{2} c_{3} A^{c} \mathscr{D}_{2}\left(s_{4}\right) \\
+\sigma^{B} \frac{\alpha_{s}^{2}\left(\mu_{R}^{2}\right)}{\pi^{2}} C_{D_{1}}^{(2)} \mathscr{D}_{1}\left(s_{4}\right)+\frac{\alpha_{s}^{d_{\alpha_{s}}+2}\left(\mu_{R}^{2}\right)}{\pi^{2}}\left\{\left(2 c_{2}-\frac{\beta_{0}}{2}\right) A^{c}+c_{3} T_{1}^{c}+F^{c}\right\} \mathscr{D}_{1}\left(s_{4}\right) \\
+\sigma^{B} \frac{\alpha_{s}^{2}\left(\mu_{R}^{2}\right)}{\pi^{2}} C_{D_{0}}^{(2)} \mathscr{D}_{0}\left(s_{4}\right)+\frac{\alpha_{s}^{d_{\alpha_{s}}+2}\left(\mu_{R}^{2}\right)}{\pi^{2}}\left\{\left[c_{1}-\zeta_{2} c_{3}+\frac{\beta_{0}}{4} \ln \left(\frac{\mu_{R}^{2}}{M^{2}}\right)+\frac{\beta_{0}}{4} \ln \left(\frac{M^{2}}{s}\right)\right] A^{c}\right. \\
\left.+\left(c_{2}-\frac{\beta_{0}}{2}\right) T_{1}^{c}+F^{c} \ln \left(\frac{M^{2}}{s}\right)+G^{c}\right\} \mathscr{D}_{0}\left(s_{4}\right) .
\end{aligned}
$$

Here $C_{D_{1}}^{(2)}=c_{3} c_{1}+c_{2}^{2}-\zeta_{2} c_{3}^{2}-\left(\beta_{0} / 2\right) T_{2}+\left(\beta_{0} / 4\right) c_{3} \ln \left(\mu_{R}^{2} / M^{2}\right)+c_{3} K / 2-\sum_{j}\left(\beta_{0} / 4\right) B_{j}^{(1)}, F^{c}=$ $\operatorname{tr}\left[H^{(0)}\left(\Gamma_{S}^{(1) \dagger}\right)^{2} S^{(0)}+H^{(0)} S^{(0)}\left(\Gamma_{S}^{(1)}\right)^{2}+2 H^{(0)} \Gamma_{S}^{(1) \dagger} S^{(0)} \Gamma_{S}^{(1)}\right]$, and $C_{D_{0}}^{(2)}, G^{c}$ can be found in [10] and involve two-loop [11] corrections.

The master formula for the NNNLO soft-gluon corrections is

$$
\begin{aligned}
& \hat{\sigma}^{(3)}=\sigma^{B} \frac{\alpha_{s}^{3}\left(\mu_{R}^{2}\right)}{\pi^{3}} \frac{1}{8} c_{3}^{3} \mathscr{D}_{5}\left(s_{4}\right) \\
& +\sigma^{B} \frac{\alpha_{s}^{3}\left(\mu_{R}^{2}\right)}{\pi^{3}}\left\{\frac{5}{8} c_{3}^{2} c_{2}-\frac{5}{2} c_{3} X_{3}\right\} \mathscr{D}_{4}\left(s_{4}\right)+\frac{\alpha_{s}^{d_{\alpha_{s}}+3}\left(\mu_{R}^{2}\right)}{\pi^{3}} \frac{5}{8} c_{3}^{2} A^{c} \mathscr{D}_{4}\left(s_{4}\right) \\
& +\sigma^{B} \frac{\alpha_{s}^{3}\left(\mu_{R}^{2}\right)}{\pi^{3}}\left\{c_{3} c_{2}^{2}+\frac{1}{2} c_{3}^{2} c_{1}-\zeta_{2} c_{3}^{3}+\left(\beta_{0}-4 c_{2}\right) X_{3}+2 c_{3} X_{2}-\sum_{j} C_{j} \frac{\beta_{0}^{2}}{48}\right\} \mathscr{D}_{3}\left(s_{4}\right) \\
& +\frac{\alpha_{s}^{d_{\alpha_{s}}+3}\left(\mu_{R}^{2}\right)}{\pi^{3}}\left\{\frac{1}{2} c_{3}^{2} T_{1}^{c}+\left[2 c_{3} c_{2}-\frac{\beta_{0}}{2} c_{3}-4 X_{3}\right] A^{c}+c_{3} F^{c}\right\} \mathscr{D}_{3}\left(s_{4}\right) \\
& +\sigma^{B} \frac{\alpha_{s}^{3}\left(\mu_{R}^{2}\right)}{\pi^{3}}\left\{\frac{3}{2} c_{3} c_{2} c_{1}+\frac{1}{2} c_{2}^{3}-3 \zeta_{2} c_{3}^{2} c_{2}+\frac{5}{2} \zeta_{3} c_{3}^{3}+\left(-3 c_{1}+\frac{27}{2} \zeta_{2} c_{3}\right) X_{3}+\left(3 c_{2}-\beta_{0}\right) X_{2}\right. \\
& \left.\quad-\frac{3}{2} c_{3} X_{1}-\sum C_{i} \frac{\beta_{1}}{8}+\sum C_{j} \frac{\beta_{0}}{16}\left[\beta_{0} \ln \left(\frac{\mu_{R}^{2}}{M^{2}}\right)+2 K\right]+\sum_{j} \frac{\beta_{0}^{2}}{16} B_{j}^{\prime(1)}+\sum_{j} \frac{3}{32} C_{j} \beta_{1}\right\} \mathscr{D}_{2}\left(s_{4}\right) \\
& +\frac{\alpha_{s}^{d_{\alpha_{s}}+3}\left(\mu_{R}^{2}\right)}{\pi^{3}}\left\{\left(\frac{3}{2} c_{3} c_{2}-3 X_{3}\right) T_{1}^{c}+\frac{3}{2}\left[c_{2}+c_{3} \ln \left(\frac{M^{2}}{s}\right)\right] F^{c}+\frac{3}{2} c_{3} G^{c}+\frac{1}{2} K_{3}^{c}\right.
\end{aligned}
$$




$$
\begin{aligned}
+\left[\frac{3}{2} c_{2}^{2}+\frac{3}{2} c_{3} c_{1}-3 \zeta_{2} c_{3}^{2}+3 X_{2}+\frac{\beta_{0}^{2}}{4}\right. & -\frac{3}{4} \beta_{0}\left(c_{2}-\frac{c_{3}}{2} \ln \left(\frac{\mu_{R}^{2}}{M^{2}}\right)\right) \\
& \left.\left.-\frac{3 \beta_{0}}{8} c_{3} \ln \left(\frac{M^{2}}{s}\right)\right] A^{c}\right\} \mathscr{D}_{2}\left(s_{4}\right)+\cdots
\end{aligned}
$$

where for brevity we have omitted the $\mathscr{D}_{1}\left(s_{4}\right)$ and $\mathscr{D}_{0}\left(s_{4}\right)$ terms (full expressions are provided in [10]). Here $X_{3}=\left(\beta_{0} / 12\right) c_{3}-\sum_{j} C_{j} \beta_{0} / 24, X_{2}=-\left(\beta_{0} / 4\right) T_{2}+\left(\beta_{0} / 8\right) c_{3} \ln \left(\mu_{R}^{2} / M^{2}\right)+c_{3} K / 4-$ $\sum_{j}\left(\beta_{0} / 8\right) B_{j}^{(1)}$, and $X_{1}, K_{3}^{c}$ can be found in [10].

The NNNLO soft-gluon corrections for top quark production at the Tevatron in the $q^{-} q$ channel were calculated in [10]. The corrections are small but they decrease further the scale dependence of the cross section, which is already small when the NNLO soft corrections (for which full results for both partonic channels and in two different kinematics are available [5]) are taken into account. When the NNNLO soft corrections are included, the cross section varies by only a few percent as we vary the ratio $\mu / m_{t}$ by two orders of magnitude. The theoretical prediction is in good agreement with data from the Tevatron $[12,13]$.

For charged Higgs production via $b g \rightarrow t H^{-}$the NNNLO soft corrections were found to be quite large $[6,10]$; the soft corrections through NNNLO provide an increase of over $80 \%$ over the leading order cross section at the LHC for a charged Higgs mass of $1000 \mathrm{GeV}$.

\section{References}

[1] The QCD/SM Working Group: Summary Report, hep-ph/0403100.

[2] N. Kidonakis and G. Sterman, Phys. Lett. B 387, 867 (1996); Nucl. Phys. B 505, 321 (1997) [hep-ph/9705234]; N. Kidonakis, G. Oderda, and G. Sterman, Nucl. Phys. B 531, 365 (1998) [hep-ph/9803241]; N. Kidonakis, Int. J. Mod. Phys. A 151245 (2000)[hep-ph/9902484].

[3] E. Laenen, G. Oderda, and G. Sterman, Phys. Lett. B 438, 173 (1998) [hep-ph/9806467]; N. Kidonakis and J.F. Owens, Phys. Rev. D 63, 054019 (2001) [hep-ph/0007268].

[4] N. Kidonakis, Int. J. Mod. Phys. A 19, 1793 (2004) [hep-ph/0303186]; Mod. Phys. Lett. A 19, 405 (2004) [hep-ph/0401147]; in DIS 2003, hep-ph/0306125.

[5] N. Kidonakis, Phys. Rev. D 64, 014009 (2001) [hep-ph/0010002]; Int. J. Mod. Phys. A 16, s1A, 363 (2001) [hep-ph/0009013]; N. Kidonakis and R. Vogt, Phys. Rev. D 68, 114014 (2003) [hep-ph/0308222]; Eur. Phys. J. C 33, s466 (2004) [hep-ph/0309045]; Int. J. Mod. Phys. A 20, 3171 (2005) [hep-ph/0410367].

[6] N. Kidonakis, JHEP 05, 011 (2005) [hep-ph/0412422]; in these proceedings, hep-ph/0511235.

[7] N. Kidonakis and A. Sabio Vera, JHEP 02, 027 (2004) [hep-ph/0311266]; R.J. Gonsalves, N. Kidonakis, and A. Sabio Vera, Phys. Rev. Lett. 95, 222001 (2005) [hep-ph/0507317].

[8] D. de Florian, A. Kulesza, and W. Vogelsang, hep-ph/0511205.

[9] A. Belyaev and N. Kidonakis, Phys. Rev. D 65, 037501 (2002) [hep-ph/0102072]; N. Kidonakis and A. Belyaev, JHEP 12, 004 (2003) [hep-ph/0310299].

[10] N. Kidonakis, hep-ph/0509079; in DPF 2004, hep-ph/0410116; in DIS 2005, hep-ph/0506299.

[11] N. Kidonakis, hep-ph/0208056; in DIS 2003, hep-ph/0307145.

[12] CDF Coll., hep-ex/0504053; Phys. Rev. D 72, 032002 (2005) [hep-ex/0506001].

[13] D0 Coll., hep-ex/0504043; Phys. Lett. B 626, 35 (2005) [hep-ex/0504058]; hep-ex/0505082. 